\title{
PIEZORESISTIVE SENSING OF A DIELECTRICALLY ACTUATED SILICON BAR RESONATOR
}

\author{
Dana Weinstein and Sunil A. Bhave \\ OxideMEMS Lab, Cornell University, Ithaca, New York 14853, USA
}

\begin{abstract}
This paper reports on a dielectrically actuated and piezoresistively sensed $4.41 \mathrm{GHz}$ silicon bar resonator with an electromechanical $Q\left(Q_{e m}\right)$ of 8180 . The 2-port piezoresistive transconductance measurement performed provides a promising alternative to capacitive measurement at high frequencies, where nominal and feed-through capacitance often dominates the output signal. The electromechanical $f \cdot Q$ product of the silicon-based resonator is $3.6 \times 10^{13} \mathrm{~s}^{-1}$, the highest reported to date for a silicon resonator using a direct 2-port measurement.
\end{abstract}

\section{INTRODUCTION}

Silicon-based high frequency electromechanical resonators offer low power, small footprint, high-Q solutions for a variety of applications including microprocessing, RF communications, and sensor networks. In recent years, much of MEMS resonator research has focused on different mechanisms for driving and sensing acoustic resonance at frequencies exceeding $1 \mathrm{GHz}$. Promising transduction methods include solid dielectric capacitive transduction [1,2], piezoelectric transduction [3,4], and air-gap capacitive actuation with piezoresistive sensing [5].

The authors previously demonstrated the internal dielectric transduction of a $4.51 \mathrm{GHz}$ silicon bar resonator [2]. The $3^{\text {rd }}$ and $9^{\text {th }}$ longitudinal-mode harmonics of the device were excited and detected capacitively with dielectric films sandwiched inside the resonator body at displacement nodes (Figure 1 ). The $9^{\text {th }}$ harmonic resonance at $4.51 \mathrm{GHz}$ with a mechanical quality factor of 11,200 , demonstrated a $9.8 \mathrm{~dB}$ improvement in signal strength over the $3^{\text {rd }}$ harmonic at $1.5 \mathrm{GHz}$. The resulting mechanical $f \cdot Q$ product of $5.1 \times 10^{13} \mathrm{~s}^{-1}$ is the highest measured to date in silicon. The analytical and experimental study of internal dielectric transduction performed in $[2,6]$ indicates improved transduction efficiency with increasing frequency, enabling mechanical resonance at previously unattainable frequencies.

A three-port scalar mixer measurement [7] was used to characterize the internal dielectrically transduced resonator. However, the large nominal and feed-through capacitance inherent in high-frequency capacitive sensing makes such resonators impractical in most integrated CMOS applications.

In this work, the $9^{\text {th }}$ harmonic of a longitudinal mode silicon bar resonator is detected in a two port measurement, coupling the benefits of internal dielectric capacitive actuation with the piezoresistive sensing demonstrated by van Beek et al [5].

\section{THEORY}

\section{Capacitive Actuation and Sensing}

Internal dielectric transduction employs capacitive drive and sense to excite and detect acoustic resonance. In [2], a longitudinal-mode bar resonator is driven and sensed electrostatically with thin vertical dielectric layers, as shown in Figure 1 . The resonator body is biased to $V_{D C}$, and a harmonic excitation of amplitude $v_{\text {in }}$ is applied to the drive electrode at the resonant frequency. The amplitude of vibrations of the $\mathrm{n}^{\text {th }}$ harmonic resonance is given by

$$
U_{0}=\frac{2 Q \varepsilon_{f} V_{D C} v_{i n}}{n^{2} \pi^{2} Y} \frac{L}{g^{2}}\left[\sin \left(k_{n} d-\frac{k_{n} g}{2}\right)-\sin \left(k_{n} d+\frac{k_{n} g}{2}\right)\right]
$$

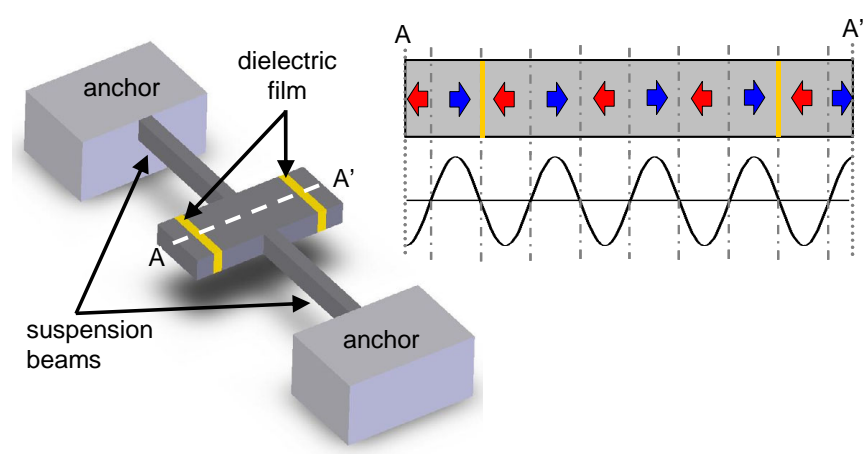

Figure 1: Schematic of internal dielectric transduced longitudinalmode bar resonator. The dielectric films (yellow) are incorporated into the resonant mode shape. A cross section of the bar along AA' is illustrated on the right. The 9th harmonic longitudinal mode is shown, with dielectric films for driving resonance positioned at displacement nodes.

where $Q$ is the quality factor, and $Y$ and $\rho$ are the Young's modulus and mass density of the resonator, respectively. Here, $\varepsilon_{f}$ is the dielectric permittivity, $g$ is the dielectric thickness, $d$ is the position of the dielectric along the bar, $A$ is the transduction area, and $k_{n}=n \pi / L$ is the resonance wave number. The 2-port motional impedance $R_{X} \equiv v_{\text {in }} / i_{\text {out }}$ for the $n^{\text {th }}$ harmonic of the resonator is given by

$$
R_{X}=\frac{n \pi \sqrt{Y \rho}}{2 Q A \varepsilon_{f}{ }^{2} V_{D C}{ }^{2}} \frac{g^{4}}{\cos ^{2}\left(k_{n} d\right) \sin ^{2}\left(k_{n} g / 2\right)}
$$

The quartic dependence of the motional impedance on dielectric thickness requires the thinnest dielectric possible, limited by fabrication and material properties. As the resonator scales to higher frequencies and the acoustic half-wavelength $\lambda / 2 \rightarrow g$, the $\sin ^{2}$ term in the denominator approaches unity, reducing motional impedance. Internal transduction incorporates the dielectric film into the resonant mode shape, resulting in increased transduction efficiency without degradation of the $f \cdot Q$ product as frequency extends into the $\mathrm{GHz}$ range.

\section{Capacitive Actuation and Piezoresistive Sensing}

Capacitive sensing at $\mathrm{GHz}$ frequencies is challenging due to large nominal and feed-through capacitance intrinsic to the device; three-port scalar-mixer measurements are often required. To overcome this obstacle, piezoresistive sensing of a capacitively actuated resonator is implemented. There are several benefits to piezoresistive detection. Geometric and frequency scaling are considerably more favorable than in the case of capacitive sensing. Additionally, independent control of the drain current enables us to set the piezoresistive transconductance $g_{m}$ to be as large as possible. When a drain current $I_{d}$ flows through a mechanically resonating structure, the piezoresistive change in the resistance $d R / R$ generates a transconductance

$$
g_{m}=\frac{d R}{R} \frac{I_{d}}{v_{i n}}
$$


where $v_{i n}$ is the AC voltage across the dielectric films capacitively exciting resonance. The change in the resistance due to piezoresistivity follows

$$
\frac{d R}{R}=\pi_{t} \sigma_{t}+\pi_{l} \sigma_{l}
$$

where $\pi_{t}$ and $\pi_{l}$ are the transverse and longitudinal piezoresistive coefficients, and $\sigma_{t}$ and $\sigma_{l}$ are the transverse and longitudinal stresses, respectively. The stress is defined by the resonant mode shape with amplitude of vibrations given by equation 1 . Summing equation 4 over all points in the resonator, weighting each point by the ratio of total current density at that point to the total current density, the total fractional piezoresistive change is given by

$$
\frac{d R}{R}=\frac{\sum_{i} j_{i}\left(\pi_{t i} \sigma_{t i}+\pi_{l i} \sigma_{l i}\right)}{\sum_{i} j_{i}} .
$$

In the case of the dielectrically actuated longitudinal-mode resonator, acoustic waves travel perpendicular to the drain current, as shown in Figure 2. Figure 3 presents the analytical results of the contribution to the piezoresistive signal distributed over the resonator body, as defined by equation 5 . The symmetry of the resonant mode and uniformity of the current flow result in a cancellation of most of the signal. With this geometry, the primary contribution to the output signal comes from vibrations in and near the suspension beams.

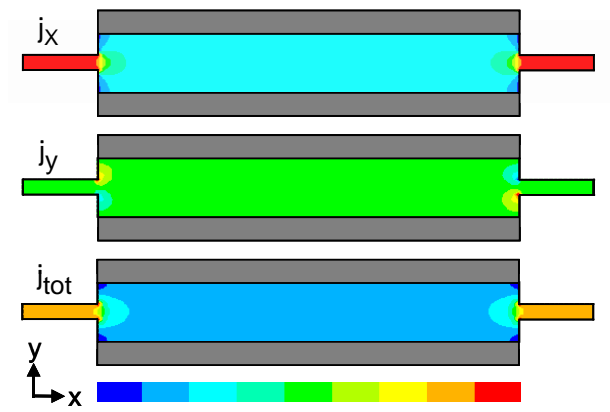

Figure 2: Contour plot of current density in the resonator. Current flow is uniform through most of the resonator body. Current nonuniformity at the input and output of the resonator is the primary source of piezoresistive signal.

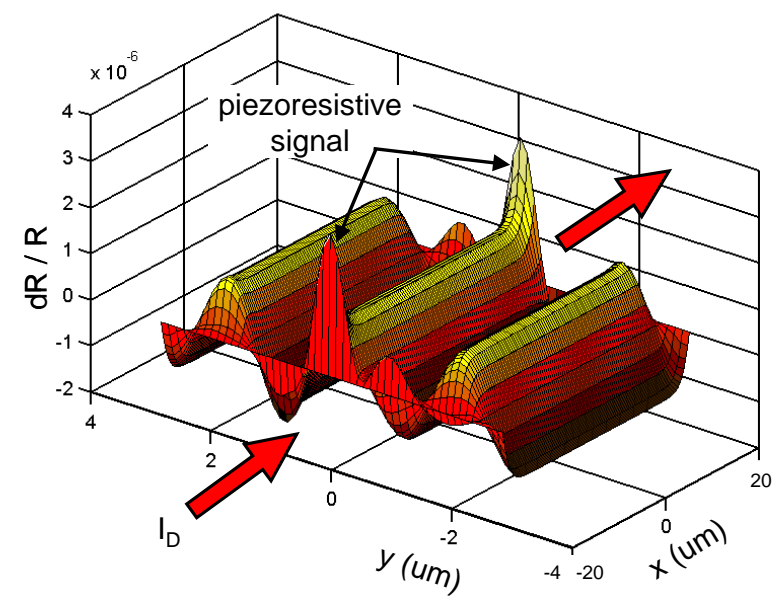

Figure 3: Spatial distribution of piezoresistive signal. The symmetry of the strain in the resonator results in cancellation of the signal in the majority of the body. The piezoresistance peaks near the routing beams generate the detected resonant signal.

\section{CALIBRATION AND MEASUREMENT}

The resonator was fabricated as described in [2], in a combined SOI-polysilicon process using a $15 \mathrm{~nm}$ silicon nitride film for dielectric actuation. Figure 4 shows a scanning electron micrograph of the $8.5 \mu \mathrm{m}$ long $\times 2.5 \mu \mathrm{m}$ thick $\times 40 \mu \mathrm{m}$ wide silicon bar resonator, with a $9^{\text {th }}$ harmonic longitudinal resonance at 4.5 GHz.

The resonator was tested at room temperature in a vacuum probe station. A schematic of the experimental setup is shown in Figure 5. A short-open-load-through calibration on a ceramic substrate was first performed to remove parasitics up to the probe tips, followed by de-embedding using short, open, and through structures on-chip. Both ends of the resonator were biased to a $V_{G}$ of $10 \mathrm{~V}$, and a $0 \mathrm{dBm}(0.2 \mathrm{~V}) \mathrm{AC}$ excitation from an Agilent parametric network analyzer (PNA) was superimposed to capacitively generate resonance. A drain current across the resonator, defined by a bias voltage $V_{d}$, was modulated piezoresistively and detected by the PNA. The transconductance is given by

$$
g_{m}=Y_{21}-Y_{12} \text {. }
$$

This technique is identical to microwave frequency measurement of the transconductance in transistors.

\section{EXPERIMENTAL RESULTS}

The measured frequency response of the $9^{\text {th }}$ harmonic longitudinal mode with drain currents of $1.22,11.82$, and $23 \mu \mathrm{A}$ is presented in Figure 6. At $23 \mu \mathrm{A}$, a resonant frequency of $4.41 \mathrm{GHz}$ with an electromechanical $Q$ of 8180 is observed with a piezoresistive transconductance of $1.1 \mu \mathrm{A} / \mathrm{V}$. The power dissipated in the resonator is $0.46 \mathrm{~mW}$.

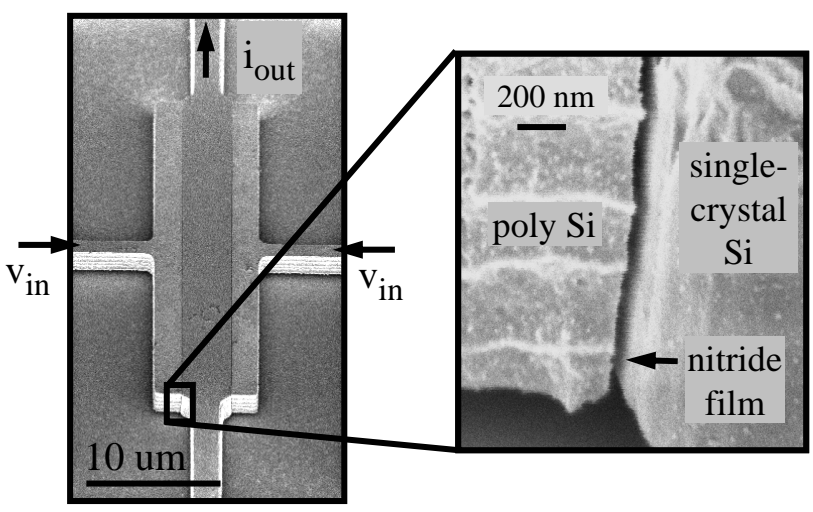

Figure 4: SEM of silicon bar resonator: $8.5 \mu \mathrm{m}$ long $\times 2.5 \mu \mathrm{m}$ thick $\times 40 \mu \mathrm{m}$ wide. An input AC voltage $v_{\text {in }}$ superimposed on a bias voltage $V_{G}$ applied to both ends of the bar drives resonance, while the output current $i_{\text {out }}$ is detected across the center of the bar. The inset image shows the thin nitride gap between the polysilicon and single crystal regions of the resonator.

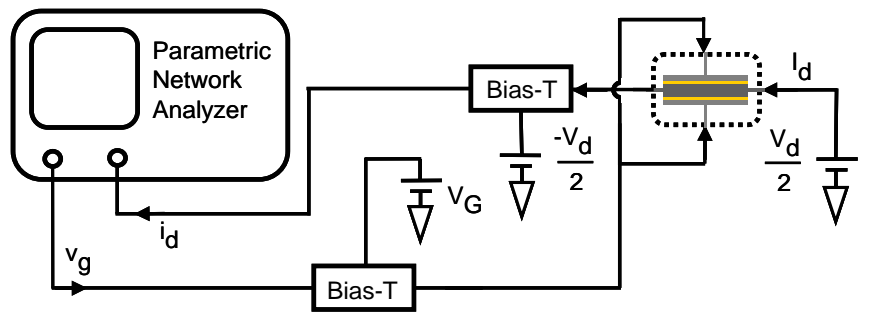

Figure 5: Schematic of measurement setup for internal dielectric actuation and piezoresistive detection. 
Several trends occur as the drain current through the resonator increases. As expected, the resonant frequency decreases with increasing bias current, as shown in Figures 6 and 7. This is due to thermal expansion of the silicon bar as a result of Joule heating. A $1.6 \%$ tuning is observed varying the current flow through the resonator from $1.22 \mu \mathrm{A}$ to $23 \mu \mathrm{A}$.

Figure 8 presents the experimental shift in electromechanical $Q$ as the current increases. The increasing $Q$ is a result of desorption of molecules from the surface of the resonator as the device is heated. At a maximum current of $23 \mu \mathrm{A}$, the resonant signal exhibits an electromechanical $f \cdot Q$ product of $3.6 \times 10^{13} \mathrm{~s}^{-1}$. While the three-port scalar mixer measurement of [2] provides an accurate measure of the resonator's mechanical $Q$, the quality factor obtained in the two-port piezoresistive measurement combines both mechanical and electrical contributions to $Q$, resulting in the electromechanical $Q$ commonly quoted in literature. The observed electromechanical $f \cdot Q$ product is the highest measured to date in silicon. Moreover, this $f \cdot Q$ product is in very good agreement with the theoretical upper bound on $Q$ presented in [8]. This indicates that the quality factor of the RF longitudinal bulk-mode resonator is limited by normal and Umklapp phonon scattering described by the Akheiser Effect.
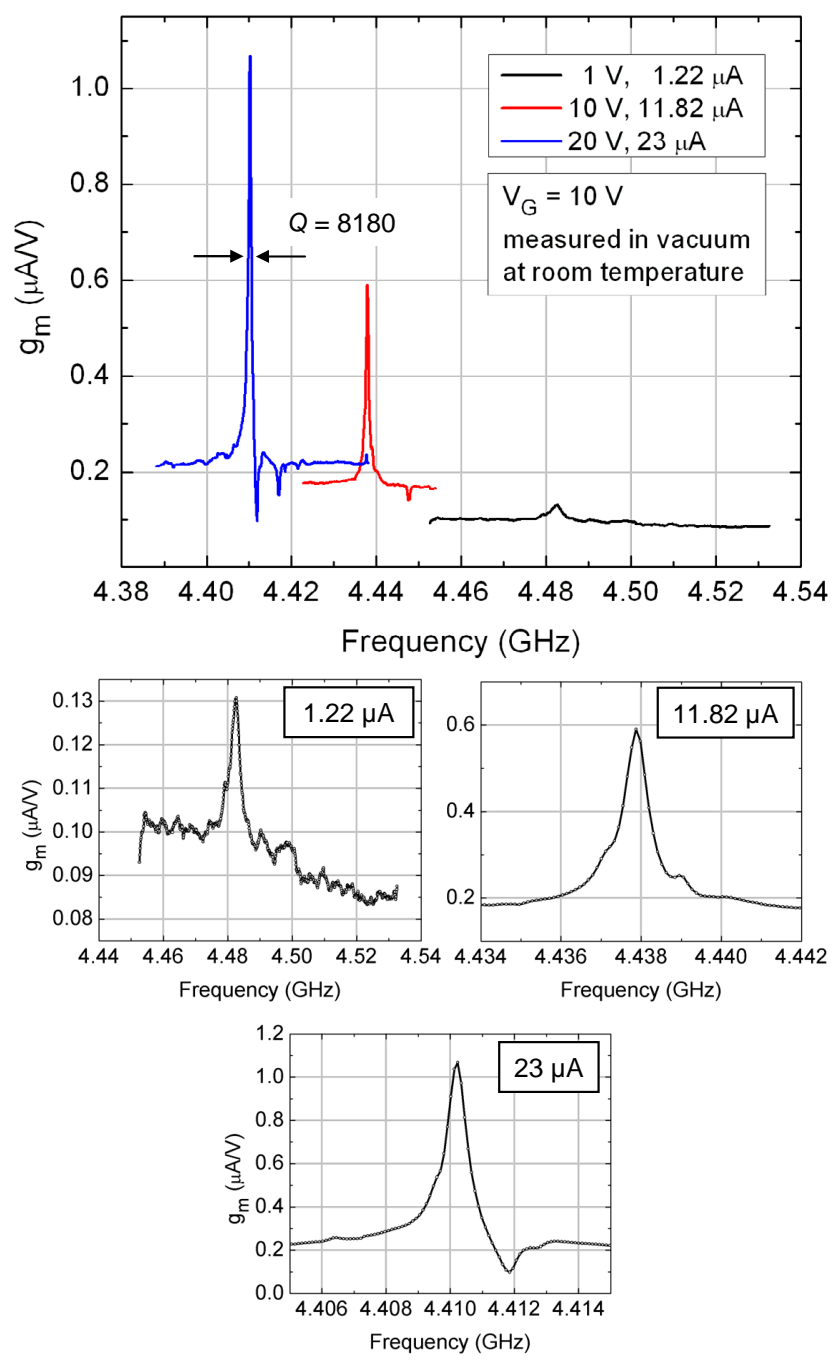

Figure 6: Measured frequency response of piezoresistive transconductance $g_{m}$ of the resonator for varying drain currents.

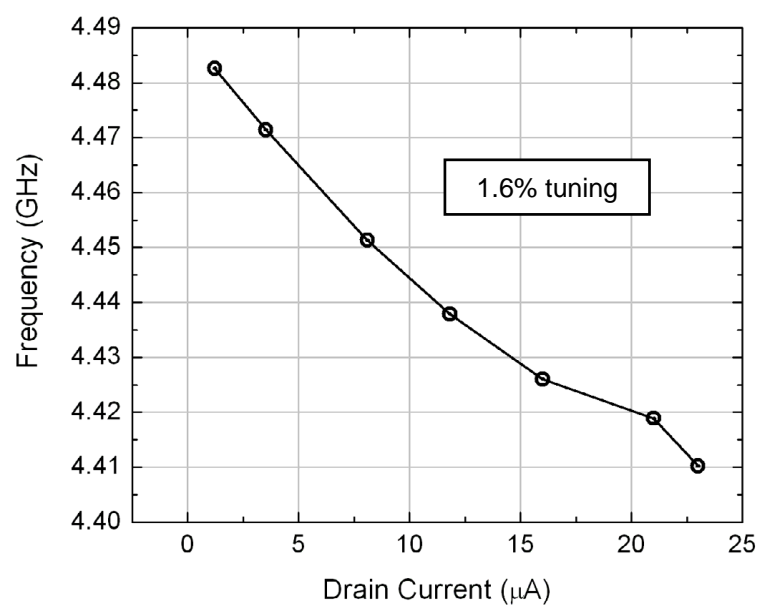

Figure 7: Measured resonant frequency scaling with increasing drain current. Resistive heating thermally expands the resonator, decreasing resonant frequency with increasing internal temperature.

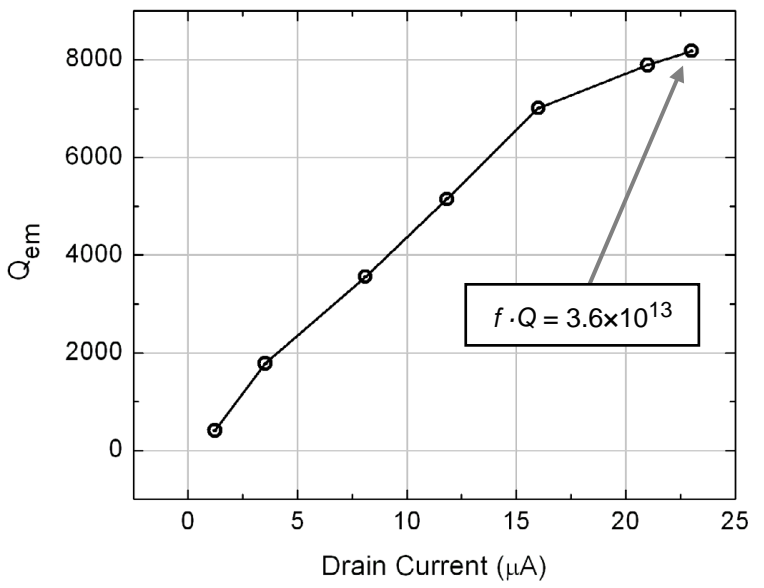

Figure 8: Measured electromechanical Q scaling with increasing drain current. The increasing trend results from desorption of molecules from the resonator surface as the device is resistively heated.

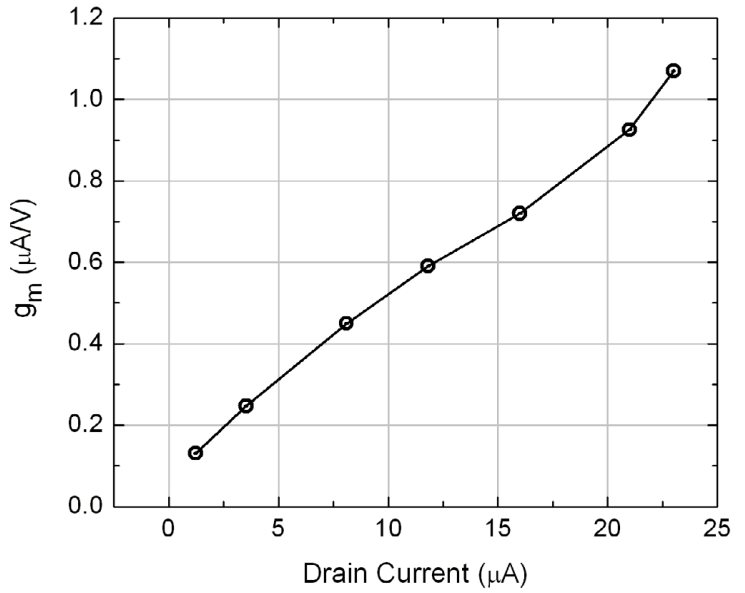

Figure 9: Experimental $g_{m}$ scaling with increasing drain current. This trend is directly dependent on $Q$, and therefore reflects the effects of surface desorption as the drain current increases and the resonator heats. 
As seen in Figure 9, piezoresistive transconductance scales linearly with drain current, dictated by equation 3 , with a $1.1 \mu \mathrm{S}$ signal at $23 \mu \mathrm{A}$ of drain current. The extracted piezoresistive $d R / R$ for this device is $1 \%$. However, the transconductance is directly proportional to the resonant $Q$, whose rising trend with increasing current flow was affected by desorption on the resonator surface. It should be noted that the measured fractional piezoresistance is therefore not indicative of equilibrium conditions.

\section{CONCLUSION}

Piezoresistive sensing was presented as a viable improvement over internal dielectric capacitive sensing in high frequency resonators, offering a two-port configuration to facilitate direct incorporation of RF MEMS resonators into integrated circuit applications. The measured resonance at $4.41 \mathrm{GHz}$ demonstrates an electromechanical $Q$ of 8180 . The resulting $f \cdot Q$ product of the resonator is higher than previously measured in silicon, indicating the promise of internal dielectric actuation in $>1 \mathrm{GHz}$ resonators. Piezoresistive sensing, which does not require a scalar mixer measurement, yields a measurement of the electromechanical $Q$ as opposed to a purely mechanical $Q$. This is the value of $Q$ used in the design of integrated devices. A low power consumption of $<1$ $\mathrm{mW}$ and control of $g_{m}$ make these NEMS-Transconductor hybrid resonators ideal candidates for integration into high-frequency CMOS technology.

\section{REFERENCES}

[1] Y.-W. Lin, S.-S. Li, Z. Ren, and C. T.-C. Nguyen, "Vibrating micromechanical resonators with solid dielectric capacitivetransducer 'gaps'," Joint IEEE Int. Frequency Control/Precision Time \& Time Interval Symposium, Vancouver, Canada, 08/29-31/05, (2005), pp. 128 - 134.
[2] D. Weinstein, S.A. Bhave, "Internal dielectric transduction of a 4.5 GHz silicon bar resonator,” IEEE International Electron Devices Meeting, Washington, DC, 12/10-12/07, (2007), pp. 415 - 418.

[3] G. Ho, R. Abdolvand and F. Ayazi, "High order composite bulk acoustic resonators," $20^{\text {th }}$ IEEE International Conference on Micro Electro Mechanical Systems, Kobe, Japan, 01/2125/07, (2007), pp. 791 - 794.

[4] G. Piazza, P. J. Stephanou and A.P. Pisano, "Single-chip multiple-frequency AIN MEMS filters based on contourmode piezoelectric resonators," Journal of Micro Electro Mechanical Systems, 16 (2), 319 - 328 (2007).

[5] J.T.M. van Beek, G.J.A.M Verheijden, G.E.J. Koops, K.L. Phan, C. van der Avoort, J. van Wingerden, D. Ernur Badaroglu, J.J.M. Bontemps, "Scalable 1.1 GHz fundamental mode piezoresistive silicon MEMS resonator," IEEE International Electron Devices Meeting, Washington, DC, 12/10-12/07, (2007), pp. 411 - 414.

[6] D. Weinstein, S.A. Bhave, "Internal dielectric transduction: optimal position and frequency scaling,” IEEE Transactions on Ultrasonics, Ferroelectrics, and Frequency Control, 54(12), 2696 - 98 (2007).

[7] A.-C. Wong, T.-C. Nguyen, "Micromechanical Mixer-Filters ('Mixlers')," Journal of Micro Electro Mechanical Systems, 13(1), 100-112 (2004).

[8] S.A. Chandorkar, M. Agarwal, R. Melamud, R.N. Candler, K.E. Goodson, T.W. Kenny, "Limits of quality factor in bulkmode micromechanical resonators,” $21^{\text {st }}$ IEEE International Conference on Micro Electro Mechanical Systems, Tuscon, Arizona, 01/13-17/08, (2008), pp.74 - 77. 